\title{
É possível avaliar um imperativo ético?
}

\author{
Is it possible to assess an ethical imperative?
}

Marco Akerman 1

Rosilda Mendes 2

Cláudia Maria Bógus 3
1 Centro de Estudos, Pesquisa e Documentação em Cidades Saudáveis (Cepedoc Cidades Saudáveis), Secretaria Municipal de Saúde de São Paulo. Av. Dr. Arnaldo 715 01246-904, São Paulo SP. akermanm.ops@terra.com.br 2 Cepedoc Cidades Saudáveis.

3 Instituto de Saúde, Secretaria Estadual de Saúde de São Paulo, Cepedoc Cidades Saudáveis.
Abstract Stakeholders and institutions involved in implementing public policies, of which health promotion is the main axis, have discussed the need to improve evaluation processes in order to appropriately understand the results of these actions. This issue has been approached with diverse emphasis - seeking elements that demonstrate the effectiveness of the actions, and developing participatory evaluation tools that address the philosophical principles of the health promotion. This article briefly presents the main current perspectives of health promotion assessment based primarily on the following references: a book published by WHO, in 2001, updates the state-of-the art in evaluation of health promotion; an initiative by PAHO implemented in the past years with an emphasis on participatory evaluation methodologies; and a project on evidence and effectiveness carried out by IUHPE, as of 2002. Finally, it addresses a set of values and principles that could guide health promotion assessment. Moreover, the paper discusses three critical variables related to developing a participatory evaluation process in a healthy city project. These perspectives demonstrate the importance of establishing a conceptual landmark for evaluation, and that health promotion assessment is not exempt from values and principles.

Key words Assessment, Health promotion, Healthy city, public policies
Resumo Na implementação de políticas públicas que têm a promoção da saúde como eixo, atores sociais e instituições têm discutido a necessidade de aprimorar processos avaliativos para conhecer, adequadamente, os resultados de tais ações. O tema tem sido abordado com ênfases distintas: buscando elementos que evidenciam a efetividade das ações, e desenvolvendo ferramentas participativas de avaliação que contemplam os princípios filosóficos do movimento da promoção da saúde. O artigo recupera as principais perspectivas quanto à avaliação em promoção da saúde tomando como principais referências: um livro publicado pela OMS em 2001 que atualiza o "estado da arte" em avaliação da promoção da saúde, uma iniciativa da OPAS desenvolvida nos últimos anos, com ênfase na avaliação participativa, e um projeto sobre evidências e efetividade da UIPES, desenvolvido a partir de 2002. Por fim, o artigo destaca um conjunto de valores e princípios que podem nortear a avaliação em promoção da saúde e, a partir da análise de uma experiência, o trabalho discute 3 variáveis críticas de um processo participativo de avaliação de um projeto de município saudável. Estas perspectivas denotam a importância de construir um marco conceitual para a avaliação em promoção da saúde que não está isenta de valores e de princípios.

Palavras-chave Avaliação, Promoção da saúde, Município saudável, Políticas públicas 


\section{Introdução}

$\mathrm{Na}$ implementação de políticas públicas que têm a promoção da saúde como eixo, atores sociais têm discutido a necessidade de aprimorar processos avaliativos para conhecer, adequadamente, os resultados de tais ações. As instituições de investigação e gestão vêm se ocupando do tema da avaliação, desenvolvendo metodologias de monitoramento e avaliação de programas, ações e atividades, para subsidiar as políticas públicas.

Destacamos que, mais que atender aos apelos legítimos de financiadores de projetos, formuladores de políticas e "tomadores" de decisão em demonstrar a efetividade de procedimentos (WHO, 1998; Buvinich, 1999; Belloni et al., 2000), também estamos preocupados em como fazer com que uma metodologia de avaliação possa contribuir para o aprendizado, a ação e a transformação de práticas sociais.

Esta discussão não é nova, e permeia a literatura de avaliação em promoção da saúde, tendo sido descrita como "um debate entre positivistas e estruturalistas" (Wimbusch \& Watson, 2000) que ainda não chegaram a nenhum consenso sobre o que é efetividade em um campo de intervenções tão complexas.

Vários autores e organismos internacionais vêm abordando o tema da avaliação em promoção da saúde com ênfases distintas: a primeira busca elementos que evidenciam a efetividade das ações, como por exemplo, os trabalhos de Davies \& MacDonald (1998), IUHPE (1999), Perkins et al. (1999), McQueen (2000; 2002), Wimbush \& Watson (2000), e outra que desenvolve ferramentas participativas de avaliação que contemplam os princípios filosóficos do movimento da promoção da saúde, como, por exemplo, os trabalhos de Wallerstein et al. (1997) e Springett (1998).

Nutbean (1998) procura evitar a polarização entre estes dois caminhos dizendo que é importante fomentar desenhos que combinem as vantagens das diferentes abordagens de investigação, de maneira que se perceba qual estará mais adequada para cada estágio do programa. A avaliação deve ser formatada de acordo com as necessidades e circunstâncias dos programas, pois nenhuma abordagem isolada será adequada para todos os tipos de programa.

\section{Perspectivas em avaliação da promoção da saúde}

A palavra perspectiva nos faz pensar em "olhares", "pontos de vista", "aquilo que se espera no futuro" ou, até mesmo, "esperança".

Olhar em perspectiva pode significar, também, ver objetos guardando-se uma certa distância. Certamente, não é a postura que adotamos neste artigo, pois o nosso envolvimento com este assunto é quase visceral e nos deixa pleno de inquietações.

Sim, vamos trazer outros olhares e pontos de vista, pois o campo está eivado de distintas concepções e práticas. Entretanto, vamos ser econômicos na apresentação desses outros olhares para não sermos redundantes.

Uma das mais recentes publicações sobre avaliação em promoção da saúde (WHO, 2001) revela no seu título - Evaluation in health promotion: principles and perspectives (Avaliação em promoção da saúde: princípios e perspectivas) - a mesma preocupação dos editores deste número da Ciência e Saúde Coletiva.

Vários dos capítulos contidos nessa publicação como, por exemplo, "A framework for health promotion evaluation" ("Um marco conceitual para a avaliação em promoção da saúde") de Rootman et al. (2001); "Beyond process and outcome evaluation: a comprehensive approach for evaluating health promotion programmes" ("Além da avaliação de processo e resultados: uma abordagem ampliada para avaliar programas de promoção da saúde") de Potvin et al. (2001) e "Evaluation in health promotion: synthesis and recommendations" ("Avaliação em promoção da saúde: síntese e recomendações") de Goodstadt et al. (2001) apresentam os principais aspectos do atual debate no campo da avaliação em promoção da saúde.

Entretanto, vale frisar, que a perspectiva ("ponto de vista") expressa no livro é, predominantemente, a dos países desenvolvidos, pois os autores envolvidos, por exemplo, no capítulo final, o de "síntese e recomendações" (Goodstadt et al., 2001) estão inseridos em organizações canadenses (Potvin et al., 2001, Goodstadt et al. 2001, Rootman, 2001), americana (McQueen \& Anderson, 2001) e inglesa (Springett, 2001).

Por outro lado, importa saber que a perspectiva desses autores sobre promoção da saúde não está guiada pelos cânones da biomedicina e que eles se guiam pelos princípios do "empoderamento", da integralidade, da partici- 
pação, da intersetorialidade, da eqüidade, da sustentabilidade e da combinação de estratégias múltiplas para promover saúde como formulação de políticas públicas, mudanças organizacionais, desenvolvimento social, leis, advocacia, educação e comunicação (Rootman, 2001).

Quanto à avaliação, a perspectiva proposta pelos idealizadores do livro é a de estimulare apoiar abordagens e práticas inovadoras de avaliação em promoção da saúde (Rootman, 2001). E para tal, os autores identificaram nove importantes dimensões que poderiam fertilizar o terreno para que iniciativas inovadoras sobre avaliação em promoção da saúde possam proliferar: 1) é um campo em evolução; 2) pode contribuir para a prática; 3) padece da falta de demonstração de evidências de efetividade de iniciativas concretas; 4) envolve uma gama ampla de abordagens e modelos; 5) oferece possibilidade de utilização de abordagens tanto quantitativas quanto qualitativas; 6) utiliza uma grande quantidade de disciplinas e abordagens da área de ciências sociais; 7) tem como base uma gama variada de modos de planejar as iniciativas; 8) requer teoria e marco conceitual para ser efetiva; e 9) propicia novos papéis para avaliadores e pesquisadores (Goodstadt et al., 2001).

Uma outra perspectiva neste campo, digna de nota, é a iniciativa da Organização PanAmericana da Saúde (OPAS) em indicar diretrizes para formuladores de política e gestores no que concerne à avaliação em promoção da saúde (PAHO, 2004a), além de desenvolver um marco conceitual e metodologias para a avaliação participativa de ações comunitárias e intersetoriais voltadas para a promoção da saúde (PAHO, 2004b).

Nessas duas abordagens, foi considerada a perspectiva de países em desenvolvimento, pois o grupo responsável pela elaboração deste material contou com representantes de países da América do Sul e Central. Sua perspectiva está calcada na advocacia de políticas públicas voltadas para a promoção da saúde e na participação ativa dos sujeitos das iniciativas no processo avaliativo.

O "Global Programme on Health Promotion Effectiveness" (Programa Global em Efetividade da Promoção da Saúde) impulsionado pela União Internacional de Promoção e Educação em Saúde (UIPES) a partir de 2002, em colaboração com a Organização Mundial da Saúde (OMS) é, também, uma outra perspecti- va sobre avaliação em promoção da saúde, que pode ser consultada na página www.iuhpe.org - clicando na "página principal" em projects. Esta iniciativa está representada em todas as regiões do mundo, sendo que a iniciativa LatinoAmericana denomina-se Proyecto sobre Evidencias de Efectividad en Promoción de la Salud. Dentre vários objetivos que se propõe atingir, a iniciativa busca demonstrar para os gestores a efetividade da promoção da saúde e para tal apoiará o desenvolvimento de projetos de avaliação neste campo. Concentra sua perspectiva, então, em construir uma base de evidências em promoção da saúde.

Ainda vale referir algumas observações importantes feitas por Restrepo (2001). Ela enfatiza que a avaliação deve ser considerada parte do planejamento das iniciativas de promoção da saúde e, assim, a prática avaliativa deve ser desenvolvida por meio de um planejamento que potencialize a participação social e a sustentabilidade das ações.

Especificamente, quanto à avaliação de projetos e iniciativas de municípios saudáveis, Werna \& Harpham (1995) já recomendavam a combinação de indicadores de processo - em especial os relacionados com os processos de participação e de fortalecimento da comunidade e das instituições - e de impacto, considerando o tempo de desenvolvimento das ações e o contexto local e a priorização dos problemas, sem sobrepor indicadores internacionais sobre aqueles gerados no nível local .

Por fim, Hartz $(1999 ; 2000)$ ressalta que o processo de avaliação envolve a coleta de dados e o processamento e análise da informação. Assim, é fundamental um planejamento adequado desde o início para que a coleta do material atenda às perguntas que se pretende responder. A avaliação supõe a utilização de um conjunto de modelos, instrumentos e técnicas. É fundamental a construção de um modelo teórico, explicitando como se espera que a política e/ou o programa exerça sua influência e a existência de dispositivos institucionais que regulamentem o processo de avaliação, além da pluralidade metodológica.

\section{Introduzindo a nossa perspectiva}

Uma perspectiva, olhar ou ponto de vista, não deve estar descolada do lugar de onde se situa "quem olha" ou "emite pontos de vista", mesmo que este olhar e opinião sejam efetuados à 
distância, como quando se olha em perspectiva quadros em uma exposição ou se analisam problemas.

Mesmo na distância, os pés do sujeito que olha algo ou, enuncia postulados, estão firmes em um determinado chão.

Os autores deste artigo enunciam sua perspectiva de um lugar social e acadêmico relacionado com a promoção da saúde: 1) estão envolvidos em uma das perspectivas, acima, enunciadas, pois são membros do grupo que elaborou e testou os materiais e recursos para avaliação participativa promovida pela OPAS (PAHO, 2004b); 2) são membros da diretoria do Cepedoc - um centro de documentação e investigação que concentra suas atividades de promoção da saúde em iniciativas de municípios saudáveis no Estado de São Paulo; 3) estiveram envolvidos nos últimos três anos com uma iniciativa concreta de avaliação participativa no município de Bertioga, SP (Akerman et al., 2002b); e 4) pesquisam outras agendas urbanas que promovam a participação social e a intersetorialidade (Mendes et al., 2002), pois entendem não ser prerrogativa do "município saudável" a contribuição exclusiva para a formulação de políticas públicas integradas, participativas, eqüitativas e sustentáveis.
Tudo que vale a pena ser feito, vale a pena ser avaliado. Por isso, compartilhamos com vários atores sociais suas indagações sobre "se funciona, ou não", o que estamos fazendo e sobre qual é o objeto da nossa atuação (Quadro 1).

Esses depoimentos reforçam a necessidade de termos de demonstrar como as iniciativas participativas e intersetoriais voltadas para a formulação de políticas públicas direcionadas para a melhoria da qualidade de vida produzem mudanças. Neste sentido, a avaliação cumpriria um papel que não é absoluto em determinar, ou não, a validade deste tipo de iniciativas, mas um eixo reflexivo sobre caminhos (Akerman et al., 2002a).

Avaliar é produzir um "juízo de valor ou mérito" para alguma ação humana sobre o ambiente social. Utiliza-se, para tal intento, um conjunto de procedimentos metodológicos. Nos primórdios, avaliadores consideravam que os fatos "falavam por si mesmos" e que a avaliação era isenta de valores. Esta teoria vem sendo cada vez mais criticada dado que os programas sociais sempre trazem valores implícitos.

O campo da promoção da saúde é exemplar neste sentido, pois está permeado de valores em disputa, e isto re-significa o processo avaliativo que contemplaria em seu desenho a explicitação de óticas, valores e princípios.

\section{Quadro 1}

Manifestações de atores sociais relacionados com objetivos e "resultados" de iniciativas de municípios saudáveis.

- Vocês acham que dizer aos prefeitos de cidades brasileiras que uma iniciativa de município saudável "empodera” a população é suficiente para eu influenciá-los a ingressar no movimento?

Depoimento do coordenador do projeto Promoção da Saúde do Ministério da Saúde em reunião realizada em 2001 com especialistas para discutir formas de advogar a iniciativa de municípios saudáveis junto com os prefeitos.

- Como saber se uma cidade está se tornando saudável? / Que resultados concretos vocês têm que podem demonstrar a melhora da qualidade de vida nas cidades onde vocês atuam?

Perguntas de jornalistas em entrevistas concedidas pela equipe do Cepedoc Cidades Saudáveis sobre as iniciativas que apoiamos.

- Desde o início eu pensei que estávamos aqui para melhorar a conscientização da população e não para resolver os problemas de esgoto da cidade.

Questionamento de um membro da Comissão Intersetorial do Projeto Bertioga Município Saudável em uma reunião de avaliação da iniciativa. 


\section{Valores e princípios que podem guiar a avaliação em promoção da saúde}

Promover saúde é promover a vida. É compartilhar possibilidades para que todos possam viver seus potenciais de forma plena. É perceber a interdependência entre indivíduos, organizações e grupos populacionais e os conflitos decorrentes desta interação. É reconhecer que a cooperação, solidariedade e transparência, como práticas sociais correntes entre sujeitos, precisam ser, urgentemente, resgatadas. Promover a saúde é uma imposição das circunstâncias atuais que apontam para a necessidade imperiosa de novos caminhos éticos para a sociedade.

Para que a promoção da saúde, então, seja praticada nessa perspectiva e não apenas como um conjunto de procedimentos que informam e capacitam indivíduos e organizações ou que controlem determinantes de grupos populacionais, a sua avaliação poderia contribuir com este caminho, e não sendo, então, um procedimento meramente técnico, explicitaria valores e princípios.

Com o intuito de aprofundar esse debate, em fevereiro de 2001, um grupo de trabalho apoiado pela Organização Pan-Americana da Saúde (OPAS), e que contou com a participação de dois dos autores deste artigo, reuniu-se em Antígua (Guatemala) e aprofundou o debate da avaliação de políticas e/ou programas de promoção da saúde. Por meio de uma metodologia participativa, o grupo pactuou um conjunto de valores e princípios norteadores da avaliação em promoção da saúde. O quadro 2 , adaptado pelos autores do presente artigo, mostra o resultado desse trabalho.

Não se pretende com os valores e princípios acima enumerados estabelecer uma "bula" para a avaliação. O "deve ser" explicitado nas condições, características condições dos princípios apontados, mais que um "dever" do processo avaliativo, é um posicionamento que delimita o "direito" dos sujeitos da avaliação.

A avaliação de um projeto ou programa, usualmente, mede, após um período de sua realização, se foram atingidos ou não os objetivos propostos e, muitas vezes, estabelece uma linha de base de medida em relação ao início do projeto ou programa. Um empreendimento social participativo e intersetorial, em que haja uma pluralidade de perspectivas e uma alta rotatividade de atores que participam, possui um "alvo móvel"; e um objetivo estabelecido, por exemplo, no começo da iniciativa, pode não estar mais ativo e em seu lugar terem surgido outros objetivos. Esta característica, também, compromete a construção de uma linha de base analítica e respectivos indicadores. Então, uma avaliação focada na análise do alcance de objetivos de médio e longo prazo pode não ser adequada, mas avaliações participativas e em tempo real poderiam minimizar esta questão.

O caso do Projeto Bertioga Município Saudável (PBMS) ilustra esta reflexão.

\section{O caso de Bertioga: um processo de avaliação e suas variáveis críticas}

O Projeto Bertioga Município Saudável (PBMS) iniciou suas atividades em março de 2000, a partir do estabelecimento do convênio de cooperação entre a Universidade de São Paulo (USP) e a Prefeitura do Município de Bertioga (Faculdade de Saúde Pública, 2000).

A estância balneária de Bertioga está localizada no litoral do Estado de São Paulo na região Administrativa da Baixada Santista da qual fazem parte outros oito municípios. De acordo com o último censo de 2000 a população possui cerca de 32.000 residentes, sendo que nos meses de dezembro a fevereiro chega a 250.000 habitantes.

O PBMS priorizou nesse primeiro ano as ações de intervenção por meio da metodologia da pesquisa-ação, técnicas participativas, visando à mobilização de atores sociais e a criação de espaços coletivos de discussão e de fomento do projeto nas diversas regiões do município.

No período de três anos, foram realizados levantamentos das lideranças locais, capacitações, oficinas de trabalho e reuniões periódicas, com o objetivo de mobilizar e envolver a sociedade civil e setores do governo, em um processo de identificação dos problemas e definição de estratégias de negociação e definição de políticas públicas locais.

Imbuídos da necessidade de buscar caminhos metodológicos para a avaliação deste contexto de ações e vontades nos deparamos com desafios críticos ao seu desenvolvimento que compartilhamos com o leitores.

\section{A primeira variável crítica}

Definição de um desenho metodológico avaliativo suficiente para captar todos os aspectos envolvidos em projetos dessa natureza, que pres- 
Quadro 2

Valores, princípios e condições para avaliação da promoção da saúde.

Valores/Princípios

Eqüidade, Justiça Social e Solidariedade

Contextualizar a iniciativa que se quer avaliar

Ter sentido prático

Oportunizar a participação como espaço para a inclusão social

Estar ancorada em múltiplos métodos

Estar comprometida com o fortalecimento de grupos sociais

Conduzir para um processo de aprendizagem dos atores sociais envolvidos

\section{Condições}

- O processo avaliativo deve revelar todas as premissas teóricas, ideológicas e políticas sobre as quais se baseia e explicitar as relações de poder existentes, incluindo aquelas que envolvem o avaliador.

- A avaliação deve levar em conta a diversidade e ser um instrumento de reflexão sobre como a iniciativa trata as iniqüidades.

- A avaliação deve respeitar e valorizar os conhecimentos e as experiências locais.

- A avaliação deve transmitir um espírito de esperança, felicidade, amor e alegria.

- O processo da avaliação deve levar em consideração o contexto local da iniciativa, inclusive as barreiras e os elementos facilitadores.

- A avaliação deve reconhecer e explicitar as diferenças das identidades culturais, sociais e econômicas entre os distintos focos da avaliação, sejam eles populações, grupos sociais, comunidades, organizações, indivíduos.

- O processo avaliativo deve ser capaz de responder: "quem", "por que" e "como".

- A avaliação deve estar integrada ao planejamento e orientada para a ação e para a mudança.

- O processo avaliativo deve contribuir para a criação de novos recursos na comunidade.

- A avaliação deve reconhecer a necessidade de criar variadas formas de divulgar resultados e de fortalecer os grupos sociais.

- O processo avaliativo deve envolver de forma apropriada aqueles que possuem interesse legítimo na iniciativa.

- A avaliação deve garantir que os grupos sociais, que estão tendo sua saúde e qualidade de vida afetados pela iniciativa, participem do processo avaliativo.

- A avaliação deve garantir que os grupos, tradicionalmente excluídos das políticas públicas, possam compartilhar e apropriar-se tanto do processo de implementação da iniciativa, quanto da avaliação propriamente dita.

- A multiplicidade de métodos deve refletir os princípios da promoção da saúde.

- A avaliação deve utilizar uma combinação equilibrada de métodos, técnicas e instrumentos qualitativos e quantitativos.

- A avaliação deve obter informação de distintas fontes de dados.

- A avaliação deve focar em estrutura, processo e resultados de curto ou longo prazo, dependendo do estágio da iniciativa.

- O processo avaliativo deve produzir indicadores que sejam oportunos e apropriados ao contexto da iniciativa.

- A avaliação deve se basear nas potencialidades da comunidade.

- A avaliação deve apoiar a solução de problemas locais.

- A avaliação deve assegurar a eqüidade, permitindo que todos os atores sociais sejam ouvidos, principalmente aqueles advindos dos grupos mais vulneráveis e com menor poder.

- A avaliação deve gerar informação que possa ser usada para a advocacia da promoção da saúde.

- A avaliação deve estimular o co-aprendizado entre atores.

- O processo avaliativo deve encorajar o diálogo e a reflexão e facilitar todas as formas de desenvolvimento do conhecimento para todos os envolvidos.

- A avaliação deve reconhecer que aprendizagem é a chave para o desenvolvimento de capacidades para os grupos e organizações locais.

- A avaliação deve conduzir para a ação e para a mudança. 
supõem a elaboração de agendas locais participativas e o fortalecimento dos sujeitos sociais.

Optou-se por um desenho metodológico que privilegiava o componente participativo. Foi realizada, permanentemente, a avaliação formativa ou de processo, que tinha por objetivo prover informações essenciais sobre o processo desenvolvido, para facilitar a introdução de mudanças durante a ação em Bertioga. Todas as atividades descritas têm registros de percepções e sugestões que foram sendo incorporadas visando solucionar problemas não previstos no planejamento e se certificar de que o caminho a ser percorrido levaria a resultados esperados inicialmente.

Assim, cada passo concluído resultou na elaboração de recomendações específicas que deveriam promover mudanças necessárias e caminhos futuros a serem perseguidos pelo projeto. Foram utilizadas técnicas participativas que favoreceram a integração das pessoas, que puderam opinar, esclarecer suas dúvidas e defender pontos de vista diferentes.

Esse tipo de avaliação formativa pode ser traduzida como um elemento motivador, no qual os envolvidos puderam acompanhar o seu próprio desenvolvimento. Trata-se de uma avaliação que "olha para frente", para correções ou adequações e procura afetar as decisões cotidianas, operativas. Portanto, esse tipo de avaliação, também chamada de avaliação contínua, é realizada durante a implantação do projeto, ou em sua fase de operação, para indicar qualitativamente o grau de mudança produzida, em relação ao processo e envolvimento dos atores (Cohen \& Franco, 1994; Contandriopoulos et al., 2000).
A esta avaliação participativa que já se desenvolvia foi incorporado o caminho implementado pela Parceria do Novo México, que publicou um manual completo para o desenvolvimento de proposta participativa em avaliação - Participatory evaluation workbook for community initiatives (Wallerstein et al., 1997; 2002).

Este material, produzido na Universidade do Novo México, foi sendo readaptado a partir da constituição de um grupo de trabalho da Organização Pan-Americana da Saúde (Washington/EUA) e se transformou em Participatory evaluation of healthy municipalities: a practical resource kit for action, finalizado em agosto de 2003, e que será aplicado por meio da realização de estudos multicêntricos em vários países das Américas, entre os quais o Brasil.

Essa avaliação participativa desenvolve-se em oito estágios que se complementam e se retroalimentam, simultaneamente: 1) compartilhar uma história comum; 2) criar uma visão comum de futuro; 3 ) identificar os diversos atores com interesse no processo avaliativo; 4) identificar metas, objetivos e indicadores; 5) identificar estratégias para se alcançar metas; 6) coletar dados e construir indicadores; 7) analisar dados; e 8) comunicar resultados (Wallerstein et al., 1997) (Figura 1).

Este processo pressupõe: um desenho compartilhado entre comunidade, técnicos e financiadores; que as habilidades e conhecimentos produzidos no desenrolar do programa sejam transferidos para os membros da comunidade; que haja contínuo processo de retroalimentação e reflexão e, finalmente, pressupõe um envolvimento de diferentes atores sociais no processo de avaliação. 


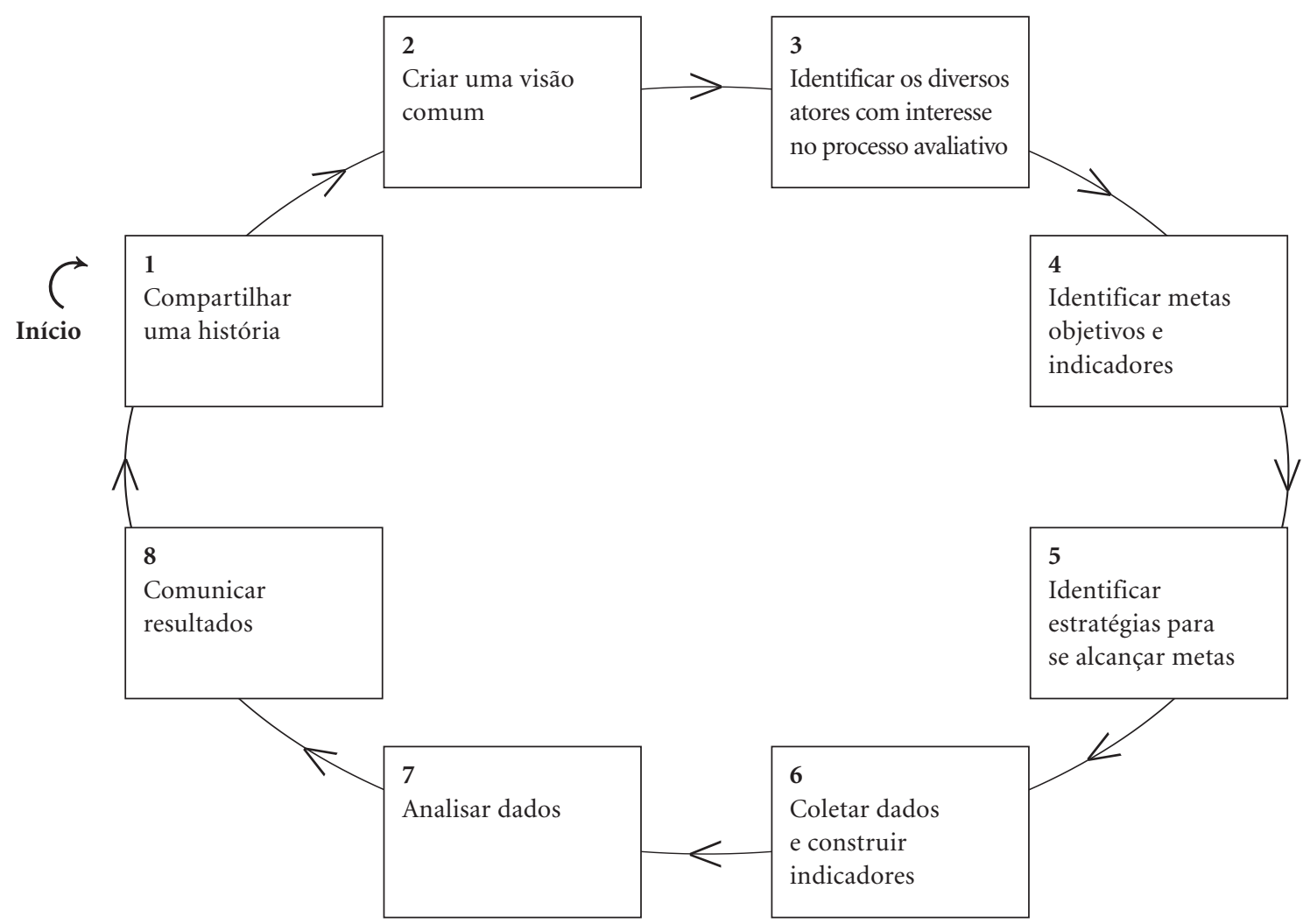

Fonte: Wallerstein N, Maltrud K \& Polacsek M. Participatory evaluation workbook for community initiatives - Healthier Communities. New Mexico - 1997.

\section{A segunda variável crítica}

Decisão quanto ao foco da avaliação, pois isto significa definir elou rever as próprias metas propostas pelos projetos.

A metodologia proposta pelo material previa, inicialmente, a definição de princípios. O grupo debateu a lista preliminarmente proposta e agregou outros princípios que representavam aspectos do contexto local.

Essa etapa foi desenvolvida por uma Comissão de Avaliação, em várias reuniões e o consenso foi facilmente alcançado. No entanto, após a definição dos princípios, iniciou-se a discussão dos objetivos e metas da avaliação, ou seja, foram formuladas "perguntas avaliativas" que poderiam permitir refletir sobre o que avaliar do processo vivenciado na iniciativa e onde chegar. Partiu-se de questões simples, que direciona- ram o "olhar avaliativo": O que é avaliar? Como chegamos até aqui? $\mathrm{O}$ que fazer para que a avaliação seja melhor? Essa discussão realizada com a Comissão Intersetorial permitiu o aprofundamento de algumas questões metodológicas, como por exemplo, a importância da definição clara e precisa dos objetivos de uma avaliação, as questões de representatividade e a explicitação dos princípios do processo avaliativo.

Esse foi o momento em que surgiram as maiores dificuldades. O grupo de atores sociais que se formou em torno da iniciativa Bertioga Município Saudável encontrou, inicialmente, dificuldades para atingir grau de consenso em relação a metas e objetivos que fossem de interesse comum.

Apesar de individualmente serem atores que já participavam de outras organizações sociais, a sua agregação em torno de um movimento, como o Projeto Município Saudável, que 


\section{Figura 2}

Modelo lógico: fortalecimento de iniciativas saudáveis.

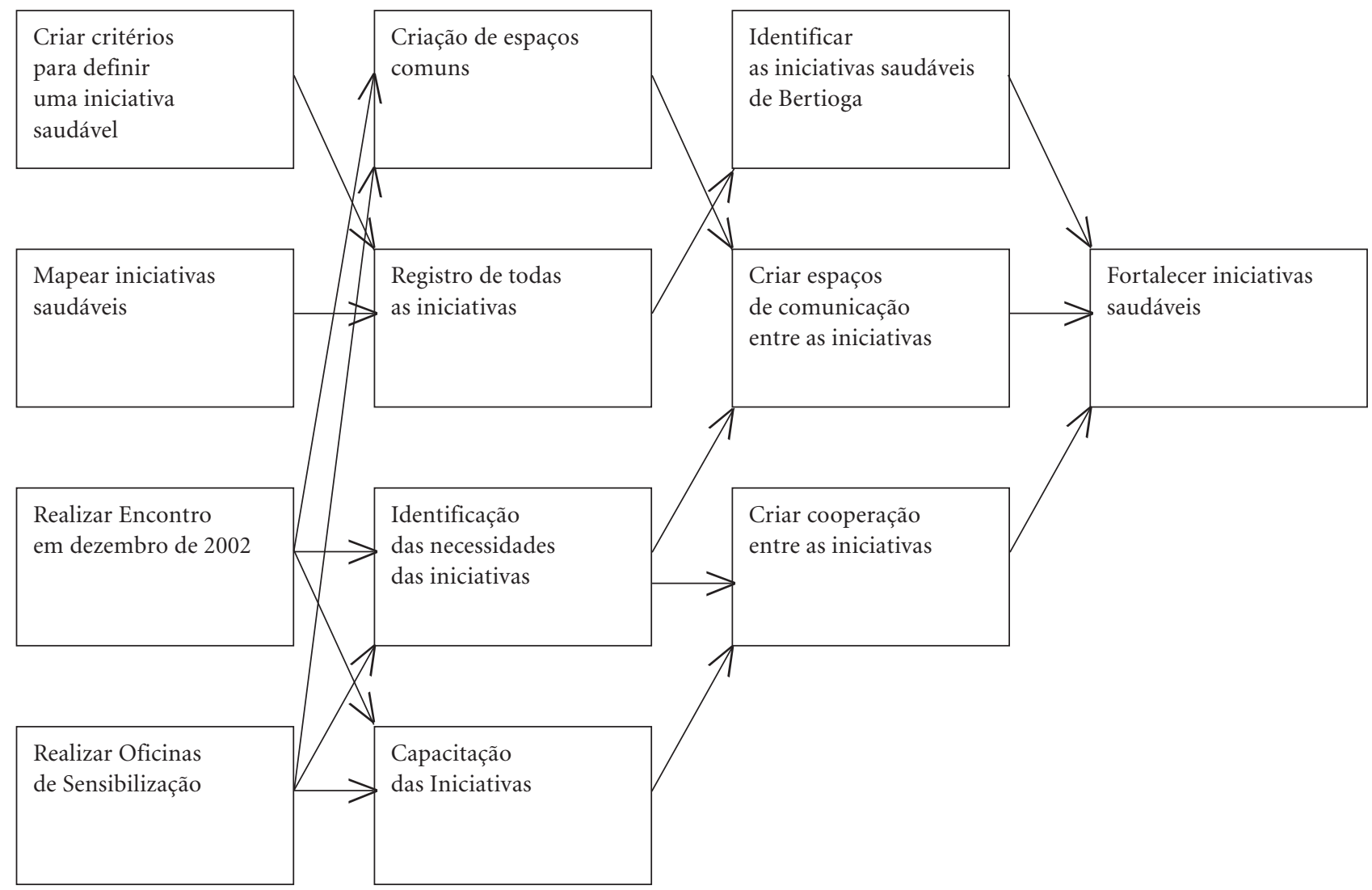

Fortalecimento

de cada Projeto

considera a cidade um todo, trouxe um grau de complexidade para a definição de metas e objetivos comuns diferentes de suas práticas vividas anteriormente, mais segmentadas e específicas dentro de territórios mais bem definidos, isto é seus bairros de origem.

Inicialmente, considerou-se que as metas e os objetivos do processo de avaliação pudessem ser definidas em função da lista de problemas existentes na saúde, na educação, na habitação, no meio-ambiente, no saneamento, etc., que foram identificados em todas as oficinas realizadas pelo PBMS. Porém, esse caminho foi infrutífero porque o grupo não conseguiu identifi- car as ações relacionadas com os problemas levantados. O que havia era a percepção desse grupo de participantes de que outras iniciativas no município poderiam fortalecer o ideário do movimento de municípios saudáveis.

As reflexões, desencadeadas no processo de avaliação, junto com a atividade de identificação de iniciativas locais que estivessem desenvolvendo ações concretas sobre problemas percebidos pela sociedade, permitiram traçar um objetivo comum: articular e apoiar a formação de uma rede social de iniciativas locais.

A citação de uma participante da população e da equipe de avaliação explicita o objetivo a ser 
avaliado: o papel do Projeto Bertioga Município Saudável em aumentar a coesão social na cidade de Bertioga. Com isto o Projeto Bertioga Município Saudável irá fortalecer e ser fortalecido por iniciativas já existentes, mas que atuam isoladamente. Estaremos fomentando estas células para que se transformem num grande organismo e esta será a nossa base para empreitadas maiores.

A formulação de um marco lógico avaliativo (Jordan, 1999; Pfeiffer, 2000), em que atividades, estratégias, objetivos e metas são explicitadas (componente cinco do "ciclo avaliativo"), foi realizada como uma possibilidade de clarear os objetivos e metas do processo avaliativo. Esperava-se que este modelo pudesse auxiliar na definição dos dados a serem coletados e dos indicadores a serem construídos e monitorados (Figura 2). Mas o marco lógico não conseguiu ser incorporado como um elemento da avaliação. Algumas hipóteses podem ser levantadas para justificar isso: ele não parece ter tido a capacidade de captar todas as dinâmicas presentes e também exigia um grande esforço de sistematização e retroalimentação da iniciativa.

\section{A terceira variável crítica}

Incorporação, na gestão, dos elementos decorrentes da prática avaliativa.

Uma pesquisa, realizada em 2002, na qual gestores de quatro cidades paulistas, incluindo Bertioga, foram entrevistados quanto à incorporação da avaliação na gestão local, apontou a desvalorização do processo em curso na cidade (Mendes et al., 2002). O depoimento de um assessor indica como o gestor entende a questão: a avaliação das políticas públicas da cidade é realizada através do prefeito, um conhecedor profundo da comunidade. Isto indica a resposta ao trabalho da administração. Ele tem claro um diagnóstico. Uma única vez que se consultou uma pessoa de fora da cidade, que utilizou um instrumento sistematizado de pesquisa, concluiu-se que a sua avaliação era igual à percepção do pre- feito (...) o prefeito é da área de saúde e sabe dos problemas da área.

Isso só reitera as dificuldades encontradas no desenvolvimento do projeto na cidade, onde o gestor local pouco se envolveu e investiu com um trabalho compartilhado de co-responsabilidade. Então, deve-se concluir que não houve nenhuma intervenção na gestão local? Ao contrário, do que possa parecer, ocorreu a elaboração de uma proposta para o Plano Diretor de Bertioga, pela comunidade envolvida no projeto, que vem sendo debatido com a Câmara Municipal (Comaru, 2004). Ou seja, o desejo por uma cidade melhor tem se manifestado na arena política.

\section{Observações finais}

O caminho percorrido pelo artigo retratou o debate corrente que se processa no campo da avaliação em promoção da saúde.

As três perspectivas internacionais assinaladas (Rootman et al., 2001; PAHO, 2004a; 2004b; IUHPE, 2002) e a perspectiva dos autores deste artigo, fundada na sua prática concreta de avaliação, representam, de alguma maneira, o debate travado na literatura e nos fóruns de promoção da saúde no Brasil e no mundo.

Estas perspectivas denotam a busca por um marco conceitual para a avaliação, a necessidade de se advogar aos "tomadores" de decisão a prática sistemática da avaliação, o uso de múltiplos métodos para a construção de uma base de evidências e os dilemas advindos de sua aplicação.

Enfatizamos que a avaliação em promoção da saúde não está isenta de valores e de princípios e que estes precisam ser explicitados pelos atores da avaliação como "roteiro" ético do processo avaliativo.

Este debate seguirá evoluindo, mas já existe um consenso: de que necessitamos, cada vez mais, de boas práticas avaliativas e de boas práticas em promoção da saúde.

\section{Agradecimentos}

Agradecemos a contribuição da professora Nina Wallerstein da Universidade do Novo México (EUA), nas discussões e na elaboração do Marco Lógico do Projeto Bertioga Município Saudável.

\section{Colaboradores}

M Akerman, R Mendes \& CM Bógus trabalharam na definição do tema e dos aspectos abordados, pesquisa bibliográfica, acompanhamento da experiência Projeto Bertioga Município Saudável, análise dos dados, redação final e na revisão do artigo 


\section{Referências bibliográficas}

Akerman M et al. 2002a. Avaliação em promoção da saúde: foco no município saudável. Revista de Saúde Pública 36(5):638-646.

Akerman M, Mendes R, Bógus CM, Westphal MF \& Wallerstein N 2002b. Desarrollo de metodología de evaluación participativa en municipiso saludables. Anais do II Congreso Chileno de Promoción de la Salud, p. 145, Santiago, Chile.

Belloni I, Magalhães H \& Sousa LC 2000. Metodologia de avaliação em políticas públicas. Cortez, São Paulo. (Coleção Questões da nossa época; v. 75).

Buvinich R 1999. A importância da avaliação de programas e projetos sociais. Fundação Banco do Brasil. Vivavoz. setembro: 8 .

Cohen E \& Franco R 2000. Avaliação de projetos sociais. 4a ed. Vozes, Petrópolis.

Comaru FA 2004. Políticas de habitação e desenvolvimento urbano em municípios saudáveis: o caso de Bertioga. Tese de doutorado. Faculdade de Saúde Pública da Universidade de São Paulo.

Contandriopoulos AP, Champagne F, Denis JL \& Pineault R 2000. A avaliação na área da saúde: conceitos e métodos, pp. 29-47. In ZMA Hartz (org.). Avaliação em saúde: dos modelos conceituais à prática na análise da implantação de programas. 1a reimpressão. Fiocruz, Rio de Janeiro.

Davies JK \& MacDonald G 1998. Evidence and effectiveness in health promotion: striving for certainties, Routledge, Londres.

Faculdade de Saúde Pública da Universidade de São Paulo e Prefeitura Municipal de Bertioga 2000. Projeto Bertioga Município Saudável. Relatório Técnico.

Goodstadt M et al. 2001. Evaluation in health promotion: synthesis and recommendations, pp. 517-533. In WHO-Europe Evaluation in Health Promotion: principles and perspectives. WHO-Europe, Copenhague.

Hartz ZMA 1999. Avaliação dos programas de saúde: perspectivas teórico-metodológicas e políticas institucionais. Ciência \& Saúde Coletiva 4(2):341-353.

Hartz ZMA 2000. Explorando novos caminhos na pesquisa avaliativa das ações de saúde, pp.19-28. In ZMA Hartz. (org.). Avaliação em saúde: dos modelos conceituais à prática na análise da implantação de programas. 1a reimpressão. Fiocruz, Rio de Janeiro.

[IUHPE] International Union for Health Promotion and Education 1999. The Evidence of Health Promotion Effectivenesss. ECSC-EC-EAEC, Luxemburgo.

[IUHPE] International Union for Health Promotion and Education 2002. Global Programme on Health Promotion Effectiveness. Disponível em <www.iuhpe.org >

Jordan G 1999. The state of the art of logic modeling. American Evaluation Association Conference.

McQueen D 2000. Strenthening the Evidence Base For Health Promotion. A Report on Evidence for the Fifthh Global Conference on Health Promotion. Méxic (DF): WHO.

McQueen D 2002. The evidence debate. Journal of Epidemiological Community Health 56(2):83-84.
Mendes R, Bógus CM \& Akerman M 2002. A percepção dos gestores municipais sobre projetos de melhoria de qualidade de vida nas cidades. Anais da $3 \underline{a}$ Conferência Regional Latino-Americana de Promoção da Saúde e Educação para a Saúde. São Paulo.

Nutbean D 1998. Evaluating health promotion - progress, problems and solutions. Health Promotion International 13(1):27-44.

[PAHO] Pan-American Health Organization 2004a. Health Promotion evaluation: Recommendations for Policymakers in the Americas. Washington DC (no prelo).

[PAHO] Pan-American Health Organization 2004b. Paticipatory Evaluation of Healthy Municipalities: a practical resource kit for acction. Washington DC (no prelo).

Perkings ER, Sinmett I \& Wright L 1999. Evidence-based health promotion. Wiley, Inglaterra.

Pfeifer P 2000. O quadro lógico: um método para planejar e gerenciar mudanças. Revista do Serviço Público 51(1):81-123.

Potvin L, Haddad S \& Frohlich KL 2001. Beyond process and outcome evaluation: a comprehensive approach for evaluating health promotion programmes, pp. 45-62. In WHO-Europe Evaluation in Health Promotion: principles and perspectives. WHO-Europe, Copenhagen.

Restrepo H 2001. Generalidades sobre evaluación de experiencias y proyectos de promoción de la salud, pp. 212-217. In H Restrepo \& H Málaga (orgs.). Promoción de la salud: cómo construir vida saludable. Editorial Médica Panamericana. Bogotá.

Rootman I, Goodstadt M, Potvin L \& Springett J 2001. A framework for health promotion evaluation, pp. 738. In WHO-Europe Evaluation in Health Promotion: principles and perspectives. WHO-Europe, Copenhague.

Springett J 1998. Practical Guidance on Evaluationg Health Promotion. WHO-Europe, Copenhague.

Wallerstein N, Maltrud K \& Polacseck M 1997. Participatory Evaluation Workbook for Community Initiatives. New Mexico Department of Health, Public Health Division, Healthier Communities Unit.

Wallerstein N, Maltrud K \& Polacseck M 2002. Participatory evaluation model for coalitions: the development of system indicators. Health Promotion Practice 3(3):361-373.

Werna E \& Harpham T 1995. The evaluation of healthy city projects in developing countries. Habitat International. 19(3):1-13.

[WHO] WHO Regional Office for Europe. 1998. Health Promotion Evaluation: recommendations to policymakers. Copenhagen.

[WHO] WHO Regional Office for Europe. 2001. Evaluation in Health Promotion: principles and perspectives. WHO-Europe. Copenhague.

Wimbusch E \& Watson J 2000. An evaluation framework for health promotion: theory, quality and effectiveness. Evaluation 6(3):301-321.

Artigo apresentado em 24/4/2004

Aprovado em 20/06/2004

Versão final apresentada em 26/6/2004 